\title{
eJRIEPS
}

Ejournal de la recherche sur l'intervention en éducation physique et sport

$26 \mid 2012$

Varia

\section{Constructivisme et pédagogie dans l'enseignement de la natation}

\section{Richard Light et Yannick Lémonie}

\section{OpenEdition}

\section{Journals}

Édition électronique

URL : http://journals.openedition.org/ejrieps/3331

DOI : 10.4000/ejrieps.3331

ISSN : 2105-0821

Éditeur

ELLIADD

\section{Référence électronique}

Richard Light et Yannick Lémonie, « Constructivisme et pédagogie dans l'enseignement de la natation », eJRIEPS [En ligne], 26 | 2012, mis en ligne le 01 avril 2012, consulté le 15 janvier 2020. URL : http://journals.openedition.org/ejrieps/3331; DOI : 10.4000/ejrieps.3331

\section{(c) (1)}

La revue eJRIEPS est mise à disposition selon les termes de la Creative Commons Attribution 4.0 International License. 
Richard Light* ${ }^{*}$ et Yannick Lémonie**

* Université de Ballarat, Australie

** Conservatoire National des Arts et Métiers, France

\section{Résumé}

Cette contribution souhaite faire le point sur la possible contribution du constructivisme au renouvellement d'une pédagogie de la natation. Si les pédagogies orientées par le constructivisme ont profondément renouvelé les manières d'appréhender l'enseignement des sports collectifs, il n'en va pas de même en ce qui concerne l'enseignement des disciplines sportives à dominantes techniques. En identifiant les différences et les points communs aux diverses formes de constructivisme, l'article propose un cadre pédagogique orienté par cette épistémologie susceptible d'être mobilisé dans l'enseignement de la natation. Deux exemples et leurs conséquences pour l'apprentissage sont proposés dans l'enseignement des techniques en papillon et en brasse.

\section{Introduction}

Les perspectives et idées constructivistes à propos ou sur l'apprentissage ont constitué une influence majeure sur la manière de penser l'enseignement et l'éducation dans les trente à quarante dernières années (Fox, 2001). Comme le notent à ce propos AmadeEscot et O'Sullivan (2007, p.186) « les théories constructivistes sont au cœur de la pensée éducative ». Le constructivisme ne se présente toutefois pas comme une théorie ou une méthode d'enseignement, mais comme une théorie de l'apprentissage (c'est d'ailleurs pourquoi nous parlerons de "pédagogie orientée par le constructivisme" et non de "pédagogie constructiviste ") : l'accent porte ainsi davantage sur les apprentissages des élèves que sur le comportement ou l'activité de l'enseignant. Néanmoins, comparé à des approches traditionnelles et prescriptives inspirées du béhaviorisme, le constructivisme suggère une approche radicalement différente de l'enseignement (Fosnot, 1996; Light, 2008).

Initialement appliquées dans le cadre de l'enseignement des mathématiques (pour une perspective francophone, voir par exemple Vergnaud, 1990), les perspectives constructivistes sur l'apprentissage ont, dans le cadre de contributions plus récentes, 
eJRIEPS 26 avril 2012

contribué à reformater les curriculums scolaires dans l'enseignement de l'éducation physique. Ainsi, si l'influence du constructivisme est évidente dans les approches popularisées dans les années 60 (Rink, 2001), leur contribution a été davantage marquante à partir des années 1990. Cette montée en puissance de la pensée constructiviste dans la manière de penser l'enseignement est en partie due à un regain d'intérêt lié à l'approche "Teaching Games for Understanding " (TGfU) dans ces années au cours desquelles les chercheurs ont tenté d'identifier en quoi les explications constructivistes sur l'apprentissage étaient consistantes avec les apprentissages générés par cette approche de l'enseignement des jeux sportifs (Kirk \& Macdonald, 1998). C'est également dans ces mêmes années que des chercheurs français ont argumenté l'intérêt de mobiliser les perspectives constructivistes pour repenser l'enseignement de l'éducation physique (Gréhaigne \& Godbout, 1998).

Le constructivisme, auquel nous associons également les idées de Lave et Wenger (1991) sur l'apprentissage situé, a donc significativement influencé la manière de penser l'apprentissage et la pédagogie dans l'enseignement des jeux (par exemple: Kirk \& Macdonald, 1998; Richard \& Wallian, 2005; Gréhaigne, Richard \& Griffin, 2005). Toutefois, cette contribution du constructivisme a été moins marquée en ce qui concerne sa mobilisation pour orienter l'enseignement et comprendre l'apprentissage au-delà des jeux et des sports collectifs. Les recherches menées dans ce domaine suggèrent pourtant que le constructivisme constitue une voie intéressante et productive susceptible d'orienter l'enseignement d'autres activités sportives enseignées dans le cadre de l'éducation physique comme par exemple, la création en danse (Chen, 2001), l'éducation du mouvement à l'école primaire (Rovegno \& Chen, 2000), ou encore de l'athlétisme (Light, 2008). Les écrits et les recherches sur la natation en France (e.g. Catteau A., 2002; Catteau R. 2008; Refuggi, 1998) suggèrent également, à travers leurs références ${ }^{1}$, que les idées constructivistes peuvent orienter l'enseignement et les recherches sur l'apprentissage en natation sportive. Toutefois, cet apport potentiel du constructivisme à l'enseignement de la natation reste à l'heure actuelle une dimension négligée dans la littérature anglo-saxonne à quelques exceptions près (e.g. Light \& Wallian, 2008; Light, 2010).

Dans la continuité de ces travaux conduits en France sur l'apprentissage et l'enseignement de la natation ancrés dans une perspective constructiviste, cet article

\footnotetext{
${ }^{1}$ L'article de R. Catteau (1981) à propos des apports de Piaget à la pédagogie en constitue une preuve convaincante.
} 
eJRIEPS 26 avril 2012

propose un cadre pédagogique souple pour un enseignement de la natation orienté par le constructivisme.

\section{Les idées constructivistes sur l'apprentissage}

\section{1. Diversité des constructivismes}

Les perspectives constructivistes sur l'apprentissage rejettent l'idée d'une réalité objective, et définissent en ce sens l'apprentissage comme un processus interprétatif façonné par l'expérience et les connaissances à partir desquelles les apprenants construisent leur propre version de la réalité (Davis, Sumara \& Luce-Kapler, 2000). Toutefois, au-delà de cette unité apparente dans la conception de l'apprentissage, le constructivisme recouvre une diversité de théories qui, bien que partageant quelques principes communs, peuvent apparaître contradictoires (Davis et al. 2000). Ces formes variées de constructivismes peuvent être décrites à partir d'une opposition entre un constructivisme cognitif, psychologique et individuel et un constructivisme socio-culturel/social (Davis \& Sumara, 2003; Fosnot, 1996; Phillips, 1997).

Le constructivisme psychologique se définit sur la base des travaux de Piaget, développés par la suite par plusieurs théoriciens ayant eu une influence dans le champ éducatif comme Von Glasersfeld (e.g. 1995). Les idées de Piaget sur l'apprentissage se sont développées à partir de ses travaux en biologie de l'adaptation et peuvent de ce point de vue être qualifiées de néo-darwiniste (Bronckart, 1999). Dans le cadre de «l'école genevoise ", l'apprentissage est conçu comme un processus à travers lequel un acteur construit activement de nouvelles connaissances à partir de ses expériences passées dans un processus de rééquilibration cognitive à la suite d'une perturbation (Cobb, 1996, p. 38). Ce constructivisme cognitif se centre plus particulièrement sur la qualité de l'interprétation individuelle et le développement des connaissances, en regardant l'apprentissage comme un processus essentiellement individuel (Cobb 1996.).

Le constructivisme social (ou socioconstructivisme) adopte un point de vue plus macro pour envisager l'apprentissage comme un processus social et interprétatif. Basé sur les idées et le travail de Vygotsky (1934), mais développé également par les travaux ultérieurs de Bruner (1966), il a été également influencé par les écrits d'autres auteurs comme Dewey. Cette forme de constructivisme questionne la centration quasi-exclusive sur la cognition individuelle du constructivisme cognitif pour proposer l'idée que l'apprentissage est culturellement et socialement situé dans un cadre plus large d'activité.

Ainsi, si les deux approches soulignent l'importance de l'expérience et de l'activité, des 
eJRIEPS 26 avril 2012

différences notables persistent, par exemple à propos de la question de savoir si la connaissance se construit au niveau individuel ou est socialement distribuée (Cobb, 1996; Davis \& Sumara, 2003).

Ces orientations disparates peuvent toutefois être vues comme ayant plus de points communs que de réelles divergences, mais la diversité des formes de constructivismes peut apparaître également problématique (voir par exemple, Cobb, 1996; Davis \& Sumara, 2003). Plusieurs auteurs ont néanmoins tenté de circonscrire ces difficultés en suggérant un certain nombre de principes que l'ensemble des approches constructivistes partage dans le cadre de l'éducation (voir par exemple Fosnot, 1996) et dans celui plus spécifique de l'éducation physique (Rovegno \& Dolly, 2006). Davis et Sumara (2003) ont ainsi réalisé ce travail en s'appuyant sur ce qu'ils considèrent comme les trois principes au cœur du constructivisme dans la formation d'une théorie de l'apprentissage complexe (Complex Learning Theory - CLT). Élaborées dans ce cadre, les premières suggestions pour les recherches sur l'enseignement de l'éducation physique en 2008 (Light, 2008) ont depuis gagné une certaine reconnaissance dans la littérature en éducation physique (e.g. Jess, Atencio, \& Thorburn, 2011). Cette CLT ne se présente ainsi pas comme une alternative au constructivisme, mais tente de contourner quelques contradictions au cœur de la diversité des approches constructivistes de manière à orienter utilement l'enseignement de l'éducation physique (Light, 2008; Jess et al., 2011).

2. 2. La théorie de l'apprentissage complexe

La CLT identifie trois principes au cœur du constructivisme compatible avec les théories de la complexité. Ces principes sont les suivants :

L'apprentissage est un processus d'adaptation: fortement influencée par les idées de Piaget, la CLT adopte une définition néo-darwinienne de l'apprentissage en le définissant comme un processus d'adaptation et de transformation. Dans ce cadre, l'apprentissage est vu comme un processus continu et complexe de transformation qui prend place dans le cadre « d'un paysage d'activité en évolution » (Davis \& Sumara, 2003, p. 125).

L'apprentissage est un processus social: la CLT reconnaît la nature sociale de l'apprentissage et de la cognition et la manière dont la connaissance se co-construit au sein des interactions sociales. D'après cette perspective, la cognition et l'esprit sont localisés au sein de ce que Saito décrit comme "un large cadre comprenant le milieu historique et socio-culturel dans lequel les êtres humains vivent »(Saito, 1996, p.400).

L'apprentissage est un processus holistique : la CLT rejette une conception réaliste de la cognition et l'apprentissage comme une transmission de connaissances. Elle envisage au 


\section{eJRIEPS 26 avril 2012}

contraire l'apprentissage comme un processus interprétatif sans la nécessité d'une référence à une réalité extérieure prédonnée (mais au contraire construite) et une nonséparation entre l'apprenant et ce qui est appris.

\section{L'enseignement de la natation et le constructivisme}

Dans la littérature internationale, l'intérêt pour le constructivisme en éducation physique a eu principalement tendance à se focaliser sur l'enseignement des jeux sportifs à partir de l'approche TGfU (e.g. Butler, 2006). Pour une large part, cet intérêt est lié à l'aide que le constructivisme peut apporter dans la compréhension et l'optimisation des apprentissages au sein d'environnements physiques (et sociaux) dynamiques (Light, 2009). Ainsi, l'approche TGfU et ses variations ultérieures ont attiré aussi bien l'attention des chercheurs que des enseignants (Light, 2005). Le constructivisme et ses développements comme la CLT (Davis \& Sumara, 2003) ou comme l'énactivisme (Varela Thompson \& Rosch, 1993) sont ainsi en mesure d'orienter l'enseignement des jeux sportifs collectifs. Le caractère dynamique de l'environnement ainsi que sa nature sociale justifie pleinement des approches pédagogiques telles que TGfU, «Game Sense » caractérisée par l'importance accordée à la réflexion sur l'action (on note ici la référence implicite à l'ouvrage de Piaget intitulé « réussir et comprendre »), les débats d’idées (Gréhaigne, Richard \& Griffin, 2005) et la formulation collaborative d'idées et de solutions testées et évaluées à des problèmes tactiques. L'ensemble de ces caractéristiques est ainsi congruent avec la définition d'un enseignement orienté par le constructivisme tel que le définit Fosnot (1996).

Cependant, un intérêt moins marqué pour le développement d'un enseignement orienté par le constructivisme peut être mis en évidence en ce qui concerne les sports individuels mettant l'accent sur les apprentissages techniques tels que l'athlétisme et la natation. Une des explications possibles à cette faiblesse est sans doute liée en partie au caractère stable de l'environnement dans lesquels prennent place ces activités et à l'importance accordée à la technique et à la répétition (Light \& Wallian, 2008). L'idée que le constructivisme peut être mobilisé pour proposer un enseignement renouvelé des techniques est également susceptible d'être découragé par la croyance dans une relation d'opposition entre techniques et tactiques qui domine les débats sur l'enseignement des jeux depuis les années 90 et qui continue d'influencer les débats actuels. Contrairement aux sports collectifs, dans l'enseignement d'une discipline comme la natation, il n'y a aucune remise en question de l'importance de la technique. Bien qu'il existe quelques 
eJRIEPS 26 avril 2012

considérations tactiques en relais ainsi que sur les longues distances, la majeure partie de l'enseignement se concentre sur la technique comme un aspect central, voire capital (Rusnak, 2008). Cela ne signifie cependant pas que celle-ci doit être enseignée à l'aide d'exercice ou d'instruction directe. Au-delà de perspectives théoriques qui voient dans ces formes d'instructions une forme d'illusion (Durand, 2008), on ne peut que noter l'existence de quelques zones d'ombre ou de quelques paradoxes, à l'instar de l'enseignement de la prise de décision en sport collectif, dans les approches directives de l'enseignement en ce domaine. Donnons un exemple : alors que les entraîneurs et les enseignants font effectuer des exercices pour développer les sensations, celles-ci ne peuvent être directement instruites dans la mesure ou l'enseignant ou le coach ne peut pas faire en sorte que le nageur sente l'eau d'une certaine façon. II ne peut dans ce cadre que faire vivre des expériences à partir desquelles le nageur est susceptible de ressentir quelque chose, mais ceci requiert un enseignement indirect susceptible d'être, dans ce cadre, orienté par le constructivisme.

Dans la section suivante, nous suggérons quelques caractéristiques qui pourraient constituer un cadre utile pour un enseignement renouvelé des techniques orienté par les théories constructivistes de l'apprentissage. Nous ne proposons pas ici un cadre prescriptif ou rigide, mais plutôt un cadre de pensée susceptible de constituer un outil pour l'enseignant ou l'entraîneur à partir de sept caractéristiques pédagogiques partagées par les approches centrées sur l'apprenant, et à partir des modèles de questionnement de l'enseignement des sports collectifs. Ceci est suivi par deux exemples pratiques de ce type de pédagogie dans le cadre de l'enseignement de la natation.

\section{Un cadre pédagogique pour l'enseignement de la natation}

Tout en reconnaissant des variations importantes dans les pratiques d'enseignement, au plan individuel, ou plus largement au plan international, il est possible de reconnaître un style traditionnel d'enseignement et d'entraînement en natation. Celui-ci tend à favoriser les instructions directes, à limiter les interactions entre nageurs ou entre l'enseignant et les nageurs, et à limiter au plan de la communication ces dernières interactions à des instructions. Alors que dans les groupes de jeunes nageurs, l'enseignement et l'entraînement mettent l'accent sur la technique, l'attention se déplace rapidement vers les aspects physiologiques dès que les nageurs sont engagés dans une concurrence plus sérieuse. Ceci se concrétise par un engagement lourd en termes de temps et d'énergie chez les nageurs (voir par exemple, Lang \& Light, 2010). Cette approche de 


\section{eJRIEPS 26 avril 2012}

l'enseignement renvoie à l'idée que le nageur est une « machine muette » (Light \& Fawns, 2001) et est sous-tendue par une vision objectiviste de la connaissance où l'acquisition de celle-ci relève in fine de la transmission entre l'enseignant ou l'entraîneur et le nageur. Dans le cadre de cette approche, les nageurs deviennent dépendants de l'enseignant en ce qui concerne les feedbacks et les instructions et sont en définitive découragés à devenir intellectuellement engagés dans leur discipline en restant enfermés dans une forme d'hétéronomie préjudiciable.

Les alternatives à cette approche traditionnelle de l'enseignement dans les sports collectifs, comme TGfU (UK), Game Sense (Australia) et Tactical Games (USA) ou encore la Pédagogie des Modèles de Décision Tactique (France) emploient une pédagogie centrée sur l'apprenant et basée sur une activité d'enquête de l'élève. Si ces alternatives diffèrent sur plusieurs aspects, elles partagent cependant certaines caractéristiques communes. Elles proposent toutes de (1) concevoir un environnement physique comme un outil pédagogique majeur; (2) mobiliser une forme de questionnement entre l'enseignant et l'élève pour stimuler la réflexion sur l'action ; (3) proposer un enseignement indirect, c'est-à-dire non prescriptif; (4) favoriser la communication entre élèves et entre les élèves et l'enseignant, où le langage joue un rôle central; (5) favoriser la réflexivité; (6) s'appuyer sur la résolution collective de problèmes sur la base de formulation de solutions testées et évaluées; (7) relier les problèmes et les solutions sur la base de formulation de principes, de règles d'action ou, à ce que Fosnot (1996) qualifie de « grandes idées ».

Même si l'enseignement des sports collectifs diffère largement de celui de l'enseignement de la natation, l'identification de ces sept principes est susceptible de permettre d'orienter utilement l'enseignement de la natation, comme celui d'autres disciplines sportives. Ces sept principes reflètent les perspectives constructivistes en matière d'apprentissage ainsi que les propositions de certaines propositions développées à partir du constructivisme, tels l'apprentissage situé (Lave \& Wenger, 1991), l'énactivisme (Varela, Thompson \& Rosch, 1991) ou encore CLT (Davis \& Sumara, 2003). Les approches de l'enseignement partageant la plupart de ces caractéristiques peuvent être qualifiées d'orientées par le constructivisme, avec un degré de variation qui dépend du degré de similarité entre l'enseignement et ces principes.

Le style d'enseignement proposé en natation relève dans ce cadre davantage de la découverte guidée que de la résolution de problème (Mosston \& Ashworth, 1986) dans la mesure où l'accent est mis sur la compréhension de la technique. En définitive, ce style d'enseignement n'est pas complètement guidé par les principes constructivistes qui 


\section{eJRIEPS 26 avril 2012}

mettent davantage l'accent sur des formes d'enquête ouverte relevant davantage d'une approche par résolution de problème (Fosnot, 1996; Light \& Wallian, 2008). Ce style n'en reste pas moins orienté par des idées constructivistes et concordant avec les trois principes clés identifiés par Davis et Sumara (2003) dans leur CLT. Sur le principe, cette approche de l'enseignement de la natation encourage les nageurs à comprendre pourquoi ils utilisent une technique et non pas simplement à comprendre comment les mettre en œuvre. Dans ce cadre, l'accent est mis sur la compréhension sur la base de la compréhension de principes et de règles d'action, comme dans l'approche TGfU. Toutefois, des différences notables subsistent. Alors que dans l'enseignement des sports collectifs, les principes sont reliés à la manipulation de l'espace et du temps, la technique et le détail de son exécution en natation sont associés à deux concepts fondamentaux de la natation: (1) réduire les résistances ; (2) augmenter la propulsion ${ }^{2}$. La mise en relation des techniques utilisés et de ces principes apparaît en mesure de permettre aux nageurs de développer une compréhension conceptuelle de leur natation et constitue une base de connaissance comprenant des connaissances corporelles et rationnelles, ainsi qu'une compréhension consciente développée à partir du langage. C'est à partir de ces connaissances progressivement incorporées à travers l'expérience que les nageurs sont susceptibles d'interpréter ce que l'enseignant leur dit, ce qu'ils ressentent et ce qu'ils font dans l'eau pour se développer en tant que nageur autonome capable de se poser et d'élucider des questions de leur propre chef.

Comme dans l'enseignement des sports collectifs, la relation entre le corps et l'esprit exprimée dans la relation entre le langage et l'action constitue une question cruciale, et éclaire la relation entre une connaissance en acte, incarnée et non consciente et une connaissance rationnelle, consciente et articulée (Light \& Fawns, 2003). Les enseignants conçoivent les expériences d'apprentissage en introduisant un obstacle conçu comme un problème à résoudre, en accordant du temps aux nageurs pour qu'ils puissent s'adapter sur la base d'une adaptation et d'une réflexion sur l'action. L'enseignant demande aux nageurs de réfléchir et d'interagir entre eux pour identifier les problèmes et discuter des solutions qu'ils ont mis en œuvre à partir de leur réflexion sur l'action. La séquence suivante consiste en l'évaluation de solutions, la discussion de ces solutions et le raffinement de celles-ci. Ces séquences sont mises en œuvre au sein de petits groupes qui les présentent ensuite à l'ensemble de la classe. Dans l'ensemble des discussions, les

\footnotetext{
${ }^{2}$ On retrouve ici la dialectique du corps projectile et du corps propulseur proposé par R. Catteau (2008)
} 
eJRIEPS 26 avril 2012

nageurs sont encouragés à relier les problèmes et les solutions aux principes clés de la natation : la réduction des résistances et l'amélioration de la propulsion.

Pour faciliter cet apprentissage, les exemples suivants incorporent les sept caractéristiques pédagogiques identifiées comme communes aux pédagogies des sports collectifs centrées sur l'apprenant.

4. 1. Exemple 1 : la seconde ondulation en papillon

Cet exemple ce centre sur l'amélioration de la technique impliquée dans la seconde ondulation en papillon par le développement d'une meilleure compréhension des raisons pour lesquelles elle est réalisée et comment elle s'intègre dans la course, sa fonction principale étant de fournir une poussée permettant la sortie de la tête pour la phase inspiratoire. Cet exemple est basé sur l'expérience d'enseignement du premier auteur de cet article avec un petit groupe de nageurs de compétition âgés de treize à 16 ans, dans le cadre d'une démonstration de la pédagogie que nous argumentons dans cet article à l'Université d'Été consacré à la pédagogie à Leeds Metropolitan University (UK) en 2010.

Après un échauffement, les nageurs ont été organisés en petits groupes de deux nageurs par ligne et il leur a été demandé de nager le papillon avec un seul bras en respirant sur le devant et non sur le côté. Nous leur avons demandé ensuite comment ils se sentaient en nageant dans cette situation particulière qui induisait une réduction de la propulsion et d'identifier les difficultés particulières éprouvées. La plupart des nageurs ont mentionné une difficulté pour inspirer dans la mesure où ils éprouvaient une difficulté à sortir la bouche de l'eau. Nous leur avons ensuite demandé comment ils pouvaient surmonter ce problème en les guidant pour obtenir une réponse sur l'ondulation. Nous leur avons ensuite demandé d'identifier quelle ondulation s'avérait problématique dans cette situation (il y a deux ondulations par cycle de nage en papillon). Après un échange avec l'ensemble de la classe à ce sujet, nous avons demandé à chaque doublette de nageurs de travailler ensemble pendant cinq minutes pour échanger, développer des solutions à ce problème, les tester, et évaluer quelle ondulation était la plus efficace et comment elle devait être réalisée. Nous leur avons ensuite demandé de s'enseigner réciproquement (Mosston \& Ashworth, 1986) les solutions trouvées pendant cinq minutes également. Ceci impliquait que l'un nageait pendant que l'autre guidait le travail de son binôme grâce à une observation et en formulant des commentaires et des suggestions avant d'échanger par la suite leur rôle. À la suite de cela, nous avons rassemblé l'équipe de huit nageurs pour discuter collectivement de leurs expériences et leur avons demandé de partager leurs découvertes relativement aux principes de poussée/propulsion et de réduction des 


\section{eJRIEPS 26 avril 2012}

résistances. Nous avons terminé la session en leur demandant d'effectuer un papillon en nage complète en leur demandant de se concentrer et d'adopter les changements dans leur seconde ondulation et finalement en leur demandant comment ils se sentaient après l'intervention. Cette séquence peut être complétée en 20 à 30 minutes même avec une grande classe ou un grand groupe de nageurs.

4. 2. Exemple 2 : Développer la sensation de l'eau

Cet exemple rend compte d'une observation réalisée avec un entraîneur travaillant avec des nageurs âgés de huit à douze ans dans un groupe de compétition à Sidney en Australie.

Développer les sensations est un aspect important pour nager correctement et particulièrement en brasse. Les sensations permettent au nageur une interprétation et un ajustement de son expérience kinesthésique qui implique une compréhension et un apprentissage implicite, c'est-à-dire incorporé, qui se développe au cours du temps. Toutefois, aussi important qu'apparaisse cette expérience sensible, ce "sens" représente un concept relativement vague pour l'enseignant ou l'entraîneur. À l'instar du « sens du jeu » pour les joueurs de sport collectif de haut niveau, cet aspect sensible de la natation correspond à une zone d'ombre pour les entraîneurs et les enseignants : il ne peut être développé à partir d'instructions directes et est bien souvent considéré comme une qualité innée chez de nombreux enseignants ou entraîneurs (Light \& Evans, 2010).

Si sentir ne peut pas être enseigné à l'aide d'instructions directes, les coachs conçoivent des situations dans lesquelles les nageurs éprouvent des expériences particulières à partir desquelles ils apprennent en réalisant, et dans lesquelles les sensations et l'expérience sensible sont l'aspect central de l'apprentissage. Alors que ces situations sont communément appelées des "exercices » par les enseignants, il n'implique cependant pas une simple répétition, un simple exercice de la technique. Ces situations aident à développer le sens de l'eau et sont conçues pour que les nageurs éprouvent des expériences kinesthésiques particulières à interpréter et à utiliser pour améliorer sa natation. Une de ces situations proposées dans l'enseignement de la brasse consiste à proposer aux nageurs de réaliser un mouvement de godille pour se propulser de manière à ressentir le positionnement idéal des mains et des avant-bras dans le début du mouvement de bras («front scull »). Dans le même temps, il est demandé aux nageurs de ne pas utiliser les jambes ou de façon limitée, éventuellement en leur proposant l'utilisation d'un pull-boy (une vidéo de ce type de situation peut facilement être retrouvée sur internet, par exemple : http://www.youtube.com/watch?v=XIhyw-uAXfo). Le problème à 


\section{eJRIEPS 26 avril 2012}

résoudre pour le nageur consiste alors à avancer de manière la plus efficace possible en n'utilisant que le début du mouvement de bras en brasse.

Cette situation souligne l'importance de ressentir la propulsion réalisée lors de la première partie du mouvement de bras et défie les nageurs de l'exécuter de manière efficiente de façon à compenser les contraintes imposées. Elle développe la sensibilité entre l'avantbras, la main et l'eau à travers un processus de résolution de problème explorant les différentes manières efficientes d'exécuter ces actions à partir des sensations plus que grâce à un processus cognitif conscient. Les enseignants dans ce cadre n'offrent généralement pas de conseils techniques, mais comptent plutôt sur les nageurs pour développer une sensation de godille mobilisable en brasse.

Cette situation permet ainsi une meilleure sensation du « catching » et du mouvement des mains et des avant-bras à travers l'eau. Ceci implique une forme préréfléchie de conscience qui peut être vue comme une adaptation corporelle ou une forme de réflexion en acte (Varela, Thompson \& Rosch, 1993). Typiquement, ce type de situation mise en place par l'enseignant ne va pas au-delà d'une structuration de l'expérience du nageur par l'effectuation de l'exercice. Une pédagogie orientée par le constructivisme permettrait dans ce cadre de prolonger et d'optimiser l'apprentissage. Ceci impliquerait par exemple de demander aux nageurs de réfléchir sur leur expérience pour construire une compréhension consciente susceptible d'être partagée entre pairs via le langage. Ce dialogue sert alors la maximisation de la poussée qui touche à la fois les aspects techniques de la godille, mais également les aspects sensibles liés à l'usage de cette technique. Contrairement à une idée répandue dans ce cadre, les jeunes nageurs sont capables de décrire leur expérience sensible et de la partager via le langage (e.g. Lémonie, 2009) et c'est sur cette possibilité que peut se développer un tel type de pédagogie.

\section{Discussion}

Même si le premier des exemples se centre sur l'acquisition technique et l'autre sur le développement des sensations, chacun de ces deux exemples pratiques partage les sept caractéristiques pédagogiques d'une pédagogie orientée par le constructivisme telles que nous avons pu les décrire. Ils impliquent que l'enseignant construise des situations conçues pour faciliter des apprentissages et des expériences particulières et une compréhension de sa natation par le nageur. Dans les deux cas, ceci implique d'imposer une contrainte que le nageur est amené à surmonter et qui implique une exagération 


\section{eJRIEPS 26 avril 2012}

comme le suggère un des principes de l'approche TGfU proposée par Griffin et Patton (2005). C'est ce que Davis et Sumara (1997) appellent une « enabling constraints », c'està-dire une contrainte permettant (enabling) l'apprentissage. Ceci implique également l'usage de certaines règles par l'enseignant au regard de comment un nageur peut compenser cette contrainte pour que l'enseignant puisse concevoir, structurer la situation, mais également guider et encourager un apprentissage basé sur ce type d'expérience. Les nageurs sont également encouragés à la réflexion sur cette expérience ce qui en accord avec Dewey (1916/1997) apparaît comme une seconde forme d'expérience. Ils sont questionnés individuellement et collectivement pour stimuler leur réflexion (individuelle et distribuée) et leur dialogue plutôt que questionnés sur ce qu'ils doivent faire, ce qui limite la réflexion et les interactions. Ils sont engagés comme des apprenants actifs plutôt que traités comme des récepteurs passifs d'une connaissance objective. Ils sont encouragés à former des petits groupes pour engager des débats d'idées (Gréhaigne, Richard \& Griffin, 2005), formuler des stratégies et des solutions, les tester, les évaluer et les présenter à la classe (Fosnot, 1996). Ceci implique qu'ils interprètent à partir de leurs expériences précédentes et en interagissant avec les autres pour construire et faire émerger aussi bien leur compréhension qu'une nouvelle connaissance. Ceci renvoie à une conception néo-darwinienne de l'apprentissage comme un processus de changement par adaptation suggéré par Piaget (voir par exemple, 1974) ainsi qu'à l'un des trois principes de la théorie de l'apprentissage complexe (Davis \& Sumara. 2003).

Après la mise en place de situations offrant des expériences permettant de faire émerger des problèmes particuliers, l'enseignant pose des questions qui, même si elles sont conçues pour faire émerger un apprentissage prédéterminé, peuvent être de nature très libre, comme Wright et Forrest (2007) en argumentent la nécessité dans l'enseignement des sports collectifs. Au moins au début, elles doivent être de nature ouverte (Carlsen, 1991). Les nageurs sont questionnés relativement aux concepts clés ( grandes idées ») de réduction des résistances et d'augmentation de la propulsion et des poussées. Comprendre l'importance de ces concepts et comment toute technique se réfère à eux est susceptible de permettre aux nageurs de devenir des apprenants autonomes qui comprennent pourquoi une technique est exécutée d'une certaine manière et qui développe une réflexion dans l'action.

Si les jeunes nageurs comprennent pourquoi ils exécutent une technique d'une certaine manière relativement aux concepts clés de la natation ils peuvent construire une expérience à partir de laquelle, de manière subséquente, des instructions plus complexes 


\section{eJRIEPS 26 avril 2012}

peuvent-être interprétées et adaptées à leur manière de nager comme un processus de production de significations. Par comprendre, nous désignons non pas seulement l'habileté à réfléchir et à articuler les connaissances, mais également l'idée de connaissance incarnée et son émergence. La conversation (Light \& Fawns, 2003) entre ces deux manières de connaître permet aux nageurs une réflexion en acte et après l'action de prendre cette expérience à un niveau réfléchi de conscience pour des considérations conceptuelles. Ceci contribue à développer les jeunes nageurs comme des apprenants indépendants. Ils gardent bien entendu le bénéfice de l'assistance de l'enseignant (ou du coach) et des interactions sociales avec leurs pairs dans lesquelles ils sont activement engagés au cours de l'apprentissage.

La pédagogie proposée ici engage les nageurs dans l'apprentissage et favorise leur capacité et leur inclination à interpréter, à travailler à partir de ce que l'enseignant dit, à mobiliser leur réflexivité et à devenir des apprenants autonomes. Dans l'enseignement de la natation, ceci implique davantage un style d'enseignement qui guide vers la découverte plutôt qu'un style de résolution de problème (Mosston \& Ashworth, 1986) où les élèves sont guidés vers la découverte de techniques prédéterminées, mais où l'enseignant doit être ouvert à de nouvelles idées et à de nouvelles solutions. En ce sens, la pédagogie que nous suggérons dans le cadre de cet article reflète bien des caractéristiques d'une pédagogie orientée par le constructivisme comme le suggère Fosnot (1996) et s'appuie de manière forte sur la créativité des élèves. Elle promeut des expériences et des interactions sociales dans lesquelles les apprenants sont confrontés et s'adaptent à des challenges physiques renforcés par les aspects sociaux engagés à travers la conversation entre une expérience d'un corps réflexif et la génération d'un dialogue que le corps exprime dans le discours (Light \& Fawns, 2003).

5. 1. Pensées conclusives : les implications pour la natation des jeunes

La pédagogie suggérée dans cet article exprime un écart important par rapport aux approches traditionnelles de l'enseignement et du coaching. Ceci implique des défis assez similaires à ceux auxquels doivent faire face les entraîneurs ou les enseignants engagés dans l'enseignement des sports collectifs en éducation physique et qui tentent d'intégrer l'approche TGfU (voir par exemple, Butler, 2005; Light, 2004). Transformer son enseignement, d'une approche centrée sur l'enseignant à une approche centrée sur l'élève, implique un changement de rôle de l'enseignant : en lieu et place de prescrire et de diriger les apprentissages, ce changement de rôle implique qu'il accompagne et qu'il facilite les apprentissages des élèves. Dans les activités comme la natation où il y a une 


\section{eJRIEPS 26 avril 2012}

centration forte sur la technique, cette transformation de l'enseignement représente un challenge important. Pour les enseignants ceci ne devrait pas constituer un obstacle trop difficile s'ils sont en mesure de prendre appui et tirer de leur propre expérience dans l'enseignement d'autres disciplines sportives impliquées dans les curriculums de formation en éducation physique et où la pédagogie implique une approche centrée sur l'apprenant et basée sur le questionnement. Cet obstacle se présente toutefois comme plus délicat pour les entraîneurs travaillant dans une activité caractérisée par un contrôle important de l'entraîneur reflétant une conception des athlètes considérés comme des « machines muettes » (Light \& Fawns, 2001).

L'approche suggérée dans cet article prend également plus de temps qu'une approche traditionnelle de l'enseignement, mais fonctionne comme un investissement précieux pour les élèves. Son adoption implique une vision à long terme de la part des enseignants, des entraîneurs et éventuellement des autres personnes impliquées comme les parents. Par ailleurs, nous de suggérons pas que chaque leçon doive suivre la structure de leçon que nous avons mise en avant et soulignée. Chacune des activités que nous avons proposées dans le cadre de cet article prend une vingtaine de minutes et cette approche peut également être mobilisée seulement en cas de besoin. Dans un groupe de nageurs de compétition, peut-être est-il opportun de suggérer cette forme de pédagogie en début de saison avant que l'intensité des entraînements n'augmente. Pour l'enseignement en milieu scolaire, toutefois, cette approche peut être considérée comme à la fois un bon moyen de faire construire aux élèves leur nage en même temps qu'un moyen permettant aux élèves d'apprendre à apprendre. Les jeunes nageurs qui apprennent dans cette voie développent une compréhension profonde de la technique et sont susceptibles, en devenant de véritables apprenants autonomes, d'identifier et de corriger d'éventuels problèmes techniques et d'adapter les changements dans leur technique à leur propre style. Ceci devrait être gratifiant tant pour les entraîneurs, les enseignants et les nageurs, même si cette relation plus égalitaire qui promeut l'autonomisation des nageurs peut-être vécue comme relativement inconfortable.

Les types de formations proposées dans l'entraînement auxquels les aspirants nageurs ont dû se soumettre ont pu conduire à l'abandon de la pratique du fait du caractère rébarbatif et ennuyeux du type d'enseignement proposé (voir par exemple, Gould, Feltz, Horn, \& Weiss, 1982; Lang \& Light, 2010). Ceci n'est pas simplement lié au caractère intensif de l'entraînement, mais également au fait que les approches traditionnelles ne promeuvent pas un engagement intellectuel alors même que les adolescents sont curieux 


\section{eJRIEPS 26 avril 2012}

et porteurs de questions sur ce qu'ils font. La pédagogie que nous préconisons dans cet article est susceptible de rendre la pratique de la natation plus intéressante et passionnante dans la mesure où elle implique les nageurs intellectuellement dans la compréhension du comment et du pourquoi de leurs actions. Cette pédagogie leur donne à penser avant, pendant et après l'action. Aider les nageurs à trouver dans l'apprentissage, à engager le dialogue avec leurs partenaires de ligne et avec l'enseignant ou l'entraîneur est susceptible de permettre la production d'un environnement intellectuel et social qui tranche avec des formations où les élèves n'ont qu'à reproduire, répéter et à regarder la ligne bleue du fond du bassin longueur après longueur, jour après jour. En plus d'aider à développer sa technique et à améliorer sa nage, cette forme de pédagogie concourt à lutter contre le désengagement préoccupant de la pratique en début d'adolescence (voir par exemple, Gould et al.).

Le problème principal à l'adoption de ce type de pédagogie relève en définitive du type de relation plus égalitaire qu'elle promeut entre l'enseignant et les nageurs et qui peut être vécue comme inconfortable. II est également possible que les entraîneurs et les enseignants soient mis en difficulté face au développement d'apprenants autonomes. Comme le suggèrent Lang et Light (2010) dans une étude sur des entraîneurs anglais de natation, la préférence pour un gros volume d'entraînement peut résulter d'une connaissance insuffisante de la technique de la part des entraîneurs. Bien que les entraîneurs puissent développer leur propre compréhension de la technique avec ce type de pédagogie, il reste néanmoins que la compréhension requise pour s'engager dans un dialogue productif avec les nageurs peut constituer un frein important à l'adoption d'une telle pédagogie.

Même s'il y a quelques obstacles à l'implémentation de ce type de pédagogie, nous avons mis en avant dans le cadre de cet article tous les bénéfices qu'elle permet pour les jeunes nageurs. Si cette approche est adoptée dans le cadre de la formation des entraîneurs et dans l'accompagnement des nageurs dans les plus jeunes catégories (par exemple poussin et benjamin), elle constituerait une préparation idéale pour les nageurs souhaitant s'engager dans les compétitions. L'accent moins important mis sur les aspects physiologiques et la compétition dans ces catégories d'âge est ainsi susceptible de constituer un espace utile pour engager intellectuellement les enfants dans la natation et pour développer une meilleure compréhension de la technique en promouvant un esprit ouvert et curieux. Si la construction de la formation des nageurs s'appuyait sur une pédagogie orientée par le constructivisme comme nous le suggérons, les nageurs âgés de 


\section{eJRIEPS 26 avril 2012}

14 à 15 ans pourraient s'appuyer sur une connaissance et une compréhension de la natation bien établie et réclameraient une attention moins soutenue que les plus jeunes nageurs.

\section{Bibliographie}

Amade-Escot, C. \& O'Sullivan, M. (2007). Research on content in physical education: theoretical perspectives and current debates. Physical Education and Sport Pedagogy, 12(3), 185-203.

Bronckart, J.-P. (1999). La conscience comme analyseur des épistémologies de Vygotski et Piaget. In Y. Clot (Ed.), Avec Vygotski (pp. 17-43). Paris : La dispute.

Bruner, J. S. (1966). Toward a theory of instruction. Cambridge, Mass. : Belknap Press of Harvard University.

Butler, J. I. (2006). Curriculum constructions of ability: enhancing learning through Teaching Games for Understanding (TGfU) as a curriculum model. Sport Education and Society, 11(3), 243-258.

Butler, J. (2005). TGfU pet-agogy: Old dogs, new tricks and puppy school. Physical Education and Sport Pedagogy, 10(3), 225-24.

Carlsen, W. (1991). Questioning in classrooms: a sociolinguistic perspective. Review of Educationnal Research, 61, 157-178.

Catteau, R. (2008). La natation de demain : une pédagogie de l'action. Biarritz : Atlantica.

Catteau, A. (1999). Un point de vue (didactique) sur l'enseignement de la natation. In $\mathrm{H}$. Hélal \& B. Boullé (Eds). L'enseignement de la natation (pp. 137-150). Paris: Éditions de l'INSEP.

Chen, W. (2001). Description of an expert teacher's constructivist-oriented teaching: Engaging students' critical thinking in learning creative dance, Research Quarterly for Exercise \& Sport, 72(4), 366-375.

Cobb, P. (1996) Where is the mind? A coordination of sociocultural and cognitive perspectives, in: C. T. Fosnot (ed.) Constructivism: Theory, perspectives and practice (pp. 34-52). New York \& London: Teachers College, Columbia University.

Davis, B. \& Sumara, D. (2003) Why aren't they getting this? Working through the regressive myths of constructivist pedagogy. Teaching Education 14(2), 123-140.

Davis, B., Sumara, J. \& Luce-Kapler, R. (2000) Engaging minds : Learning in a complex world. New Jersey: Lawrence Erlbaum Associates, Publishers. 
eJRIEPS 26 avril 2012

Fosnot, C. T. (1996). Constructivism: A psychological theory of learning. In C. T. Fosnot (ed.) Constructivism : Theory, perspectives and practice (pp. 103-119). New York \& London: Teachers College, Columbia University.

Gould, D., Feltz, D., Horn, T., \& Weiss, M. (1982).Reasons for attrition in competitive youth swimming. Journal of Sports Behavior 5, 155-165.

Gréhaigne, J-F. \& Godbout, P. (1998). Observation, critical thinking and transformation : Three key elements for a constructivist perspective of the learning process in team sports. In: R. Feingold, R. Rees, G. Barette, I. Fiorentino, S. Virgillio, \& E. Kowalski (eds) Education for life. New York: Adelphi University Press.

Griffin, L. L. \& Patton, K. (2005) Two decades of Teaching games for Understanding : Looking at the past, present and future. In L. L. Griffin \& J. Butler (eds.) Examining a Teaching Games for Understanding model (pp. 1-15). Champaign: Human Kinetics.

Jess, M., Atencio, M., \& Thorburn, M. (2011) : Complexity theory: supporting curriculum and pedagogy developments in Scottish physical education, Sport, Education and Society, 16(2), 179-199.

Kirk, D. \& Macdonald, D. (1998). Situated learning in physical education, Journal of Teaching in Physical Education, 17, 376-387.

Lang, M., \& Light, R. L. (2010). Interpreting the Long Term athlete Development Model: English swimming coaches' views on the (swimming) LTAD in practice. International Journal of Sports Science \& Coaching 5(3), 389-403.

Lémonie, Y. (2009). Étude de l'interaction d'enseignement-apprentissage. Le cas de l'enseignement de la natation sportive en EPS. Thèse de doctorat non publiée, Université Paris Est, Créteil.

Light, R. L. (2010). Children's social and personal development through sport: A case study of an Australia swimming club. Journal of Sport and Social Issues 34(4), 266-282.

Light, R. (2009). Understanding and enhancing learning in TGfU through complex learning theory. In T. Hopper, J. Butler, \& B. Storey (eds), TGfU...Simply good pedagogy: Understanding a complex challenge (pp. 23-34). Toronto, HPE Canada.

Light, R. (2008) Complex learning theory in physical education: An examination of its epistemology and assumptions about how we learn. Journal of Teaching in Physical Education, 27, 21-37. 
eJRIEPS 26 avril 2012

Light, R. (2004) Australian coaches' experiences of Game Sense: Opportunities and challenges. Physical Education and Sport Pedagogy, 9(2), 115-132.

Light, R. L. \& Evans, J. R. (2010) The impact of Game Sense pedagogy on elite level Australian rugby coaches' practice: A question of pedagogy. Physical Education and Sport Pedagogy, 15(2), 103-115.

Light, R. \& Fawns, R. (2001). The thinking body: Constructivist approaches to games teaching in Physical Education. Melbourne Studies in Education, 42(2), 69-87.

Light, R. \& Fawns, R. (2003). Knowing the game: Integrating speech and action through TGfU. Quest, 55(2), 161-176.

Light, R. \& Wallian, N. (2008). A constructivist approach to teaching swimming. Quest, 60(3), 387-404.

Mosston, M. \& Ashworth, S. (1986). Teaching Physical Education ( $3^{\text {rd }}$ ed.). Columbus : Merrill.

Piaget, J. (1974). La prise de conscience. Paris, PUF.

Refuggi, R. (1998). Apprendre à transformer sa nage pour mieux la connaître. Rôle des conceptions du monde physique en EPS. In C. Amade-Escot (Ed.), Didactique de l'éducation physique : états des recherches (pp. 325-338). Paris : Revues EPS.

Richard, J-F, \& Wallian, N. (2005). Emphasizing student engagement in the construction of game performance. In L. L. Griffin, \& J. I. Butler (eds) Teaching Games for Understanding: Theory, research and practice (pp. 19-32). Champaign, IL.: Human KInetics

Rink, J. (2001). Investigating the assumptions of pedagogy, Journal of Teaching in Physical Education, 20, 112-128.

Rovegno, I. (1998). The development of in-service teachers' knowledge of a constructivist approach to physical education: Teaching beyond activities, Research Quarterly for Exercise and Sport, 69(2), 147-162.

Rovegno, I. \& Chen, W. (2000). Examination of expert and novice teachers' constructivistoriented teaching practices using a movement approach to elementary physical education. Research Quarterly for Exercise \& Sport, 71(4), 357-372.

Rovegno, I. \& Dolly, P. (2006) Constructivist perspectives on learning. In D. Kirk, D. Macdonald, \& M. O'Sullivan, (eds). The handbook of physical education (pp. 242261). London, Thousand Oaks \& New Delhi: Sage.

Rusnak, J. (2008). From age group to elite. Swimming in Australia, 24(3), 42-43. 
eJRIEPS 26 avril 2012

Varela, F. J., Thompson, E. \& Rosch, E. (1993). L'inscription corporelle de l'esprit : Sciences cognitives et expérience humaine. Paris : Seuil.

Vergnaud,G. (1990). La théorie des champs conceptuels. Recherches en Didactique des Mathématiques, 10(2/3), 133-170.

Von Glasersfled, E. (1995). Radical constructivism: a way of knowing and learning. London : Falmer Press.

Wright, J., \& Forrest, G. (2007). A social semiotic analysis of knowledge construction and game centred approaches to teaching. Physical Education \& Sport Pedagogy, 12(3), 273-287. 